\title{
Editorial
}

\section{Toward Better Benchmarking}

\author{
Richard Platt, MD, MS
}

There is good news and bad news about nosocomial infections in general and surgical-site infection (SSI) surveillance in particular. The good news is the increasing attention our society is paying these problems. For example, some state legislatures are requiring hospitals to report their nosocomial infection rates; the Joint Commission on Accreditation of Healthcare Organizations (JCAHO) has developed new performance standards regarding perioperative antibiotic prophylaxis'; Medicare will begin to pay hospitals according to their performance ${ }^{2}$; and the Institute for Healthcare Improvement has targeted nosocomial infections in its effort to prevent 100,000 deaths each year. ${ }^{3}$ It is difficult to recall another time when so great an opportunity existed to make meaningful strides in preventing the burden of morbidity, mortality, and costs that these infections impose.

The bad news is that hospitals do not have important information about their own infection rates that would make the efforts noted above as effective as they should be, and prospective patients cannot know whether their risk of infection will be higher in one hospital than another because the hospitals themselves do not know how they compare with others performing the same procedures. Without that information, no one knows which hospitals should be models for good care. Much of the current research will not provide this information in the foreseeable future.

This issue of Infection Control and Hospital Epidemiology provides three examples of surveillance research addressing SSIs. ${ }^{4-6}$ Mitt et al. used a combination of telephone calls to patients, healthcare worker questionnaires, and review of outpatient medical records to assess infections following cesarean section in approximately 300 women in an Estonian teaching hospital. ${ }^{6}$ In Italy, Moro et al. conducted a 1-month survey of 6,167 procedures of many types per- formed in 31 hospitals; postdischarge infections were identified by telephone contacts and forms that patients brought with them to their postoperative medical visits. ${ }^{5}$ Geubbels et al. describe a Dutch study of nearly 14,000 hip arthroplasties performed in 36 hospitals; postdischarge surveillance was performed through registration cards completed by surgeons and optional record review by infection control practitioners. ${ }^{4}$

Although these studies had different goals, each invested a great deal of effort in surveillance systems to identify SSIs that occurred during and after hospitalization. Admirable as these efforts are, we need a different, or at least an additional, conversation about SSIs. The most important issues for us to address are (1) ensuring that clinicians and hospitals use established best practices and (2) developing postoperative SSI surveillance systems that more effectively support benchmarking and require less expenditure of scarce resources.

Regarding best practices, in most situations we should simply act on credible information in the literature rather than invest the effort required to demonstrate that it holds in additional settings. The new JCAHO measure regarding selection, timing, and duration of perioperative antibiotic prophylaxis is an important example. As an aside, that measure might be better if it allowed hospitals to vary from the standard, if they could justify their reasons for doing so. As written, the JCAHO prophylaxis standard appears not to permit a hospital with a high rate of methicillinresistant Staphylococcus aureus infection to use vancomycin routinely for prophylaxis. ${ }^{7}$ It also does not appear to allow a hospital to conduct research to identify better prophylaxis regimens. The JCAHO prophylaxis measure does, howev$\mathrm{er}$, have the advantage of focusing hospitals on ensuring the delivery of prophylaxis. 
However, care improvement efforts generally are seriously hampered because we do not know where problems exist. We do not know because we have no acceptable, widely usable method for benchmarking. Our current surveillance methods fall short in several ways. It is widely believed that you cannot manage what you cannot measure. It is also true that you cannot measure what you cannot define. The most widely used SSI definition includes several criteria, including "a surgeon's diagnosis of infection," allowing circularity that defies standardization. ${ }^{8}$ In addition, we have no efficient method of collecting the information that is available. The three studies in this issue of Infection Control and Hospital Epidemiology each used a slightly different method for identifying infections, meaning that their data could not be directly compared even if the same procedures had been studied. More importantly, even if it were possible to standardize the surveillance methods described by these and many other authors, most hospitals cannot sustain the effort as part of normal activities. Thus, the most widely used surveillance methods are best suited to support specific individual programs that allow epidemiologists with sufficient resources to develop internal standardization and quality control. Research quality surveillance systems like these are required to address specific questions, but they are not especially useful as the basis for routine evaluation of outcomes.

We need different surveillance methods to provide the routine information that hospitals need and that the public increasingly wants. Our goal should be that a hospital with a high rate of postoperative infection for a specific procedure, compared with other hospitals, know this so that it can determine whether it should take corrective action. A secondary goal should be to avoid devoting more personnel resources, already scarce, than absolutely necessary to collecting information. One way to achieve these goals may be to monitor indicators of infection that are derived from the routine delivery of healthcare, rather than infections themselves. These indicators would identify hospitals in which additional information about actual infections is needed. The Centers for Disease Control and Prevention (CDC) Prevention Epicenters recently demonstrated that antibiotic exposure was a useful indicator of SSI after several types of procedures; antibiotic exposure was reasonably specific if the antibiotics were received during a sufficiently long interval or extended to a readmission. ${ }^{9}$ Separately, it appears that insurers' and other payors' claims data can identify hospitals that have high rates of SSI after some types of procedures. Insurers' claims may be a particularly useful and efficient means for identifying indicators of infections that occur after discharge. ${ }^{10,11}$ Both of these approaches, monitoring antibiotics and using claims data, require wider testing to determine their utility, but they share the desirable characteristics of not requiring collection of new data from healthcare providers or patients for every procedure and not depending on subjective criteria. Although such measures do not directly monitor infections, their utility lies in identification of a small fraction of hospitals in which collection of more detailed information is worthwhile.

In addition to focusing routine surveillance on more accessible, less subjective indicators of SSI, we need other new tools to perform meaningful benchmarking. These include better case-mix adjustment methods so that hospitals that care for high-risk patients are not penalized in comparisons. We need methods to accommodate the many situations in which a hospital performs too few procedures of a particular type to provide stable estimates of its infection rate. We need to understand how much emphasis to put on identifying postdischarge infections. Although it is clear that a majority of SSIs become manifest after discharge and that these postdischarge infections incur substantial morbidity and cost, we do not know whether monitoring these infections is required to identify high-risk hospitals. We also need to understand how to make best use of the electronic medical records that are increasingly becoming available in both hospitals and ambulatory settings.

We have made great progress since the CDC's landmark demonstrations of the utility of SSI surveillance to identify ways that hospitals can reduce their infection rates. It is important to do the new research needed to move surveillance from a research tool to one that can be widely used to support improved care.

\section{REFERENCES}

1. Joint Commission on Accreditation of Healthcare Organizations. Surgical Infection Prevention National Quality Measures. Oakbrook Terrace, IL: Joint Commission on Accreditation of Healthcare Organizations. Available at www.jcaho.org/pms/core+measures/2zesiplist. pdf. Accessed March 13, 2005 .

2. Centers for Medicare \& Medicaid Services. Medicare "Pay for Performance (P4P)" Initiatives. Baltimore, MD: Centers for Medicare \& Medicaid Services; 2005 . Available at www.cms.hhs.gov/media/ press/release. asp? Counter=1343. Accessed March 13, 2005.

3. Institute for Healthcare Improvement. 100k Lives Campaign. Cambridge, MA: Institute for Healthcare Improvement. Available at www. ihi.org/IHI/Programs/Campaign. Accessed March 13, 2005.

4. Geubbels ELPE, Wille JC, Nagelkerke NJD, Vandenbroucke-Grauls CMJE, Grobbee DE, de Boer AS. Hospital-related determinants for surgical-site infection following hip arthroplasty. Infect Control Hosp Epidemiol 2005;26:435-441.

5. Moro ML, Morsillo F, Tangenti M, et al. Rates of surgical-site infection: an international comparison. Infect Control Hosp Epidemiol 2005;26:442-448.

6. Mitt P, Lang K, Peri A, Maimets M. Surgical-site infections following cesarean section in an Estonian university hospital: postdischarge surveillance and analysis of risk factors. Infect Control Hosp Epidemiol 2005;26:449-454.

7. Joint Commission on Accreditation of Healthcare Organizations. Surgical Infection Prevention National Quality Measures. Oakbrook Terrace, IL: Joint Commission on Accreditation of Healthcare Organizations. Available at www.jcaho.org/pms/core+measures/2zgsip2.pdf. Accessed March 11, 2005.

8. Mangram AJ, Horan TC, Pearson ML, Silver LC, Jarvis WR. Guideline for prevention of surgical site infection, 1999. Infect Control Hosp Epidemiol 1999;20:247-278.

9. Yokoe DS, Noskin GA, Cunningham MA, et al. Enhanced identification of postoperative infections among inpatients. Emerg Infect Dis 2004;10:1924-1930.

10. Platt R, Kleinman $K$, Thompson $K$, et al. Using automated health plan data to assess risk for hospital infection following coronary artery bypass surgery. Emerg Infect Dis 2002;8:1433-1441.

11. Miner AL, Sands KE, Yokoe DS, et al. Enhanced identification of postoperative infections among outpatients. Emerg Infect Dis 2004;10:19301937 